\title{
MULTI-ECHELON SUPPLY CHAIN SIMULATION USING METAMODEL
}

\author{
Laigang Song \\ Xueping Li \\ Alberto Garcia-Diaz \\ Department of Industrial and Information Engineering \\ the University of Tennessee \\ 404 East Stadium Hall \\ Knoxville, TN 37919, U.S.A.
}

\begin{abstract}
Metamodels are abstractions of the simulation model that expose the system's input-output relationship through simple mathematical expression (Santos and Santos 2007). It provides an analytical way to study the behavior of a complex system. Multi-echelon supply chain are one of the complex systems. It is hard if not impossible to draw close-form analytical solutions due to the complexities of inventory system and the underlying uncertainty. In this paper, we will apply the methodology of simulation metamodel to a multi-echelon supply chain problem and make statistically analysis of the parameters. The model is validated using training experiment conditions.
\end{abstract}

\section{INTRODUCTION}

Regardless of the complexity, most systems can be viewed as a transformation function between the input variables and the response variables. The simulation model is constructed to approximate the reality as much as possible. However, for very complex system the simulation model will be large and hard to understand. Constraints, such as cost and difficulty of the model development, can prevent the modeler from building multiple prototypes of the real system. In such case a simpler model "metamodel" is often built. The term "Metamodel" was proposed by (Kleijnen 1986). It has been widely used in simulation community to study the behavior of complex system.

Many definitions of metamodel are available. Barton defines the metamodel as a model of the simulation model and expose the fundamental relationship between the input and output. Following this definition, many famous theorems can be viewed as metamodels. For example the Little's Law $L=\lambda W$ is a metamodel of a simulation of queue (Barton 1992). R.Santos et. al. (Santos and Santos 2007) define metamodel as abstractions of the simulation model that expose the system's input-output relationship through simple mathematical expression.
Metamodels are very useful in many aspects. Firstly, they can show the basic properties of the target system. The findings from a metamodel can be used to verify and validate the model. Secondly, the metamodel can used to identify the factors which has the most significant impact of the output. Thirdly, due to the low computer resource requirement, the metamodel can be replicated for many times to test different scenarios. This is particularly important when the output is random. When the original model is a component of a very complex system, metamodel can be used to substitute the original component. In some case, it is the only solution due to the impractically slow or large original models.

Metamodels can be built in three steps:

1. Decide input value. The input values should be designed based on the principals of the design of experiments. A robust design experiment works well across a broad range of scenarios and provides solutions that are less likely to produce unexpected results (Kleijnen and van Beers 2005). The detailed input design method can be found (Kleijnen 2008).

2. Collect the response data. The resulting simulation responses are collected from the experiments.

3. Estimate the parameters. The appropriate mathematical model with few parameters will be calibrated so that the responses can be fitted.

The metamodel can be constructed from the collected data using different methods. The linear polynomial approximations are the most widely used methods due to its simplicity (Kleijnen 2007b, Kleijnen 2007a). Nonlinear techniques are more complex but more flexible and powerful. Nonlinear regression is the mode studied nonlinear techniques (Reis dos Santos and Porta Nova 2006). Other methods include Kriging (Kleijnen 2007a), neural network (Badiru and Sieger 1998), Bayesian approaches (Cheng 1999).

Supply chain is a complex system due to its complexity and uncertainty. The fierce competition in the market forces 
all practitioners pay attention to not only themself but also all entities in the supply chain. The ultimate goal is to maintain the customer relationship at a high level. The customers always expect their demand to be met as soon as possible. Good relationships between the service suppliers and the customers are built over long time cooperation. There are many criteria to measure the level of the service. Among all the criteria, the service level agreement (SLA) is the most commonly used one. The SLAs are different from each other, but they typically involve the service time limit related to a certain piece of equipment. For large companies, the vast amount of customers leads to various service levels. For small companies, the situation may not be so complex, but still the companies need to be prepared for multiple service level requirements.

To provide better customer service experience, the simplest way is to increase the on-hand inventory for potential demands. For manufacturing industries, producing more products will increase the possibility of satisfying the customers' demand instantly. For service industries, maintaining more spare parts will result in shorter service time. But the on-hand inventory level can not be too high due to the inevitable holding cost and purchasing cost. For some expensive items, having a high level of inventory means that a high amount of capital is locked by these items (Hopp and Spearman 2000). Thus, the annual revenue from the capital will be reduced. This contradiction is faced by almost each manager in the world. Specifically, the decisions faced by supply managers in procuring and positioning parts to satisfy these complex service agreements at minimum inventory investment have become particularly difficult (Caggiano et al. 2007).

Much work has been done in the inventory optimization of a multie-chelon system under the constraints of the time based service level agreements. Caggiano (Caggiano et al. 2007) investigates a continuous review inventory model for a multi-item multi-echelon service parts distribution system in which a time-based service level requirement exists. By driving exact time-based fill-rate expressions for each item within its distribution channel, an intelligent greedy algorithm for large-scale problems is proposed. The simulation results show that their algorithm is efficient to large-scale problems. Caglar (Caglar, Li, and Simchi-Levi 2004) examines a multi-item, two-echelon system with the objective of minimizing total system inventory investment subjected to an average response time constraint. They propose a method based on Lagranigian decomposition. The experiments show the method is efficient for fairly large problems. Wong et al. (Wong et al. 2007) investigate the same problem as in (Caglar, Li, and Simchi-Levi 2004). The experiments show that their greedy algorithm has better computation performance. With the help of some heuristics, their algorithm can be even more efficient.

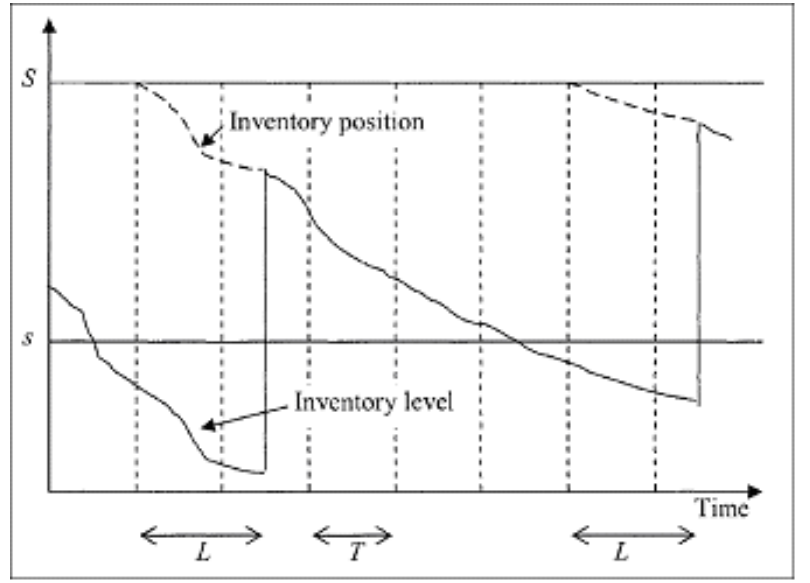

Figure 1: A periodic-review (s, S) policy with continuous demand

In this paper, a multi-echelon distribution system with time-based service level requirements is considered. The simulation model is built. We then apply the principles and techniques of metamodeling to reveal the fundamental characteristics. The remainder of the paper is organized as follows. In section 2 , the detailed description of the supply chain will be given. In section 3, the techniques of metamodel analysis will be presented. In section 4, the analysis results will be presented. The conclusion and discussion will be found in section 5 .

\section{MULTI-ECHELON SUPPLY CHAIN MODEL}

\subsection{Periodical Review $(s, S)$ Inventory Model}

The basic idea of a periodical review $(s, S)$ inventory model is very simple: If the stock level at the beginning of a period is below a certain value $s$, then order up to the level of $S$. If the initial stock level is above $s$, then no replenishment order is placed. Figure 1 shows the change of the inventory level and inventory position under periodical review $(s, S)$ policy with continuous demand where $L$ is the lead time and $T$ is the length of the review period.

It has been shown that for a single echelon system the optimal inventory policy is of the $(s, S)$ type (Tijms 1972). In this paper, the $(s, S)$ policy will be used in all locations.

\subsection{Model Assumption}

The following assumptions are considered:

1. The distribution network has an arborescent structure. That is, each location is replenished from exactly one parent node of higher echelon. There is only one location at the root of the distribu- 
Song, Li, and Garcia

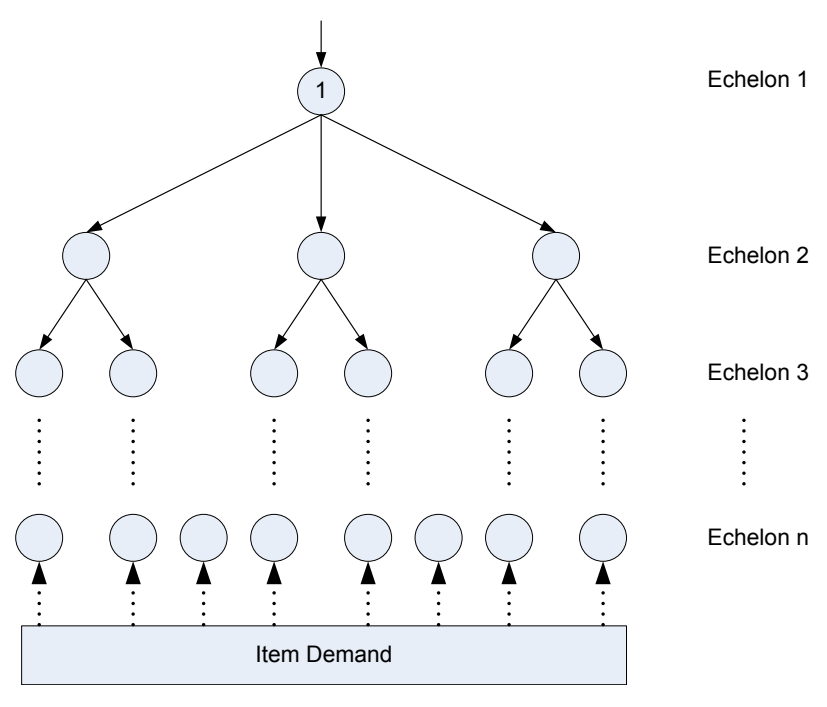

Figure 2: The arborescent structure of the distribution network

tion network. Figure 2 shows the topology of the distribution network.

2. Demands only occurs at the leaf stations in the lowest echelon. All demand locations are assumed to be in the same echelon. To achieve this property, a dummy station might be needed.

3. The demand processes at all locations are independent Poisson process and the coefficients are known and stationary.

4. The demand is for one unit of an item at one time.

5. The unsatisfied order will be backordered.

6. The demand location will serve the demand on a first-come-first-serve basis across the distribution network.

\subsection{The Decision Paradigms}

For multi-echelon inventory system research, two principal decision paradigms are used to determine the optimal base stock inventory level. The first is solely cost driven. A cost function is typically formulated during the modeling process and the optimal value is obtained by using optimal search technology. The only objective is to minimize the expected total cost. Many researches have been done using this paradigm, such as Clark and Scarf (Clark and Scarf 1960, Hadley and Whitin 1985, Axsater 2000, Zipkin 2000).

The second paradigm is the service level driven approach. In this paradigm, the constraints of meeting the customer demands within a certain probability are considered besides the cost function. Customer demands are either satisfied immediately or in a short time period. Silver (Silver, Pyke, and Peterson 1998) discusses a list of the service

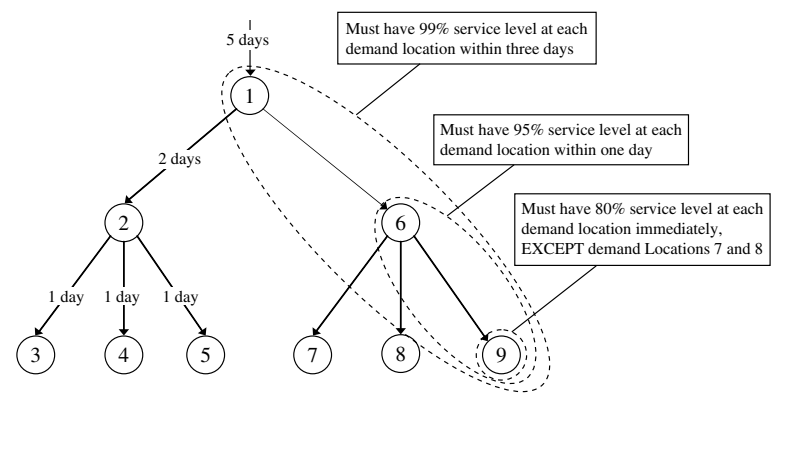

Figure 3: A multi-echelon supply chain network

level measurements. Two commonly used definitions are the probability of no shortage and the fill rate. In this paper, the service level is measured by the fill rate. The fill rate is defined as the probability that an arriving demand for an item will be satisfied within a specified period of time.

\subsection{Model Configuration}

Figure 3 shows the supply chain used in this paper.There are three echelons. Echelon 1 has only one station "1". Station 1 is replenished from echelon 0 (not shown in this figure). The lead time for the replenishment is 5 days. Echelon 2 has two stations "2" and "6". They are replenished from station 1. The lead time between echelon 1 and 2 is 2 days. Echelon 3 has 6 stations. Stations "3", "4" and "5" are replenished by station "2"; stations "7", "8" and "9" are replenished by station "6". The lead time between echelon 2 and echelon 3 is 1 day. The service level requirements are different among different demand stations. Demand stations " 3 ", "4", "5", and "9" require $80 \%$ immediately service level, $95 \%$ service level within one day and $99 \%$ service level within 3 days. Demand stations "7", "8" have the same one-day and three-day requirements, but no requirement on immediate service. When the demand can not be satisfied immediately, we impose a much higher shortage cost. We are interested in how to reduce the average total cost.

$$
C_{\text {Avg Total }}=C_{\text {Avg ordering }}+C_{\text {Avg shortage }}
$$

\section{METAMODEL ANALYSIS}

The mathematical representation of a simulation's inputoutput function can be written as

$$
\bar{Y}=f(\bar{X})
$$

where $\bar{Y}, \bar{X}$ are vector values and will usually contain random components. In our simulation model, the vector $\bar{X}$ will 
include the demand rates, $s$ and $S$ for each station and the initial inventory for each station. The lead time and the transportation time might also be included. $\bar{Y}$ could be the average total cost, average holding time, average shortage cost and so on. The response vector $\bar{Y}$ might have more than values, but we typically focus our attention on only one components of $\bar{Y}$. If so, Equation (2) can be written as

$$
y=f(\bar{X})+\varepsilon .
$$

Then the task of metamodeling is to find a good approximation of function $f$ and the model of $\varepsilon$. The major issues in metamodeling includes i) the choice of a functional form for $f$, ii) the design of experiments, i.e.. the selection of a set of $x$ points at which to observe $y$ (run the full model), the assignment of random number streams, the length of runs, etc., and iii) the assessment of the adequacy of the fitted metamodel (confidence intervals, hypothesis tests, lack of fit and other model diagnostics). The functional form will generally be described as a linear combination of basis functions from a parametric family. So there are choices for families (e.g., polynomials, sine functions, piecewise polynomials, wavelets, etc.) and choices for the way to pick the representation horn within a family (e.g. least squares, maximum likelihood, cross validation, etc.). The issues of experiment design and metamodel assessment are related since the selection of an experiment design will be determined in part by its effect on assessment issues (Barton 1992).

\subsection{Mathematical Model}

As stated previously, metamodel is used to find the inputoutput relationship. Given this purpose, the inputs are carefully designed based on some principles. For details of the design of the input value please refer to (Kleijnen 2008). The experiments are carried based on the designed input values. The output data are collected as following:

$$
Y_{i j k}: i=1, \ldots, N ; j=1, \ldots, R_{i} ; k=1, \ldots, K_{i j}
$$

where $N$ is the number of experiment conditions. $R_{i}$ is the number of replications of experiment condition $i . K_{i j}$ is the total number of observation for experiment condition $i$, replication $j$.

The mean response for each replication is

$$
\bar{Y}_{i j .}=\sum_{k=1}^{K_{i j}} \frac{Y_{i j k}}{K_{i j}}, i=1, \ldots, N, j=1, \ldots, R_{i}
$$

The central limit theory indicates that when the sample size is large, the output approximates normal distribution.

$$
\bar{Y}_{i j .} \sim N\left(\mu_{i}, \sigma_{i}^{2}\right)
$$

If the target response is the sum or average of certain observations, the target can be modeled using normal distribution based on the Central Limit Theorem. Normal distribution can be adequately described by its mean and variance. Now the parameters $\mu_{i}, \sigma_{i}^{2}$ needs to be estimated based on the collected data.

Many statistical methods are available to estimate the parameters, but most of them are based on the assumption that the observation are collected from independent and identical distribution (iid). One common technique is to test the following hypothesis

$$
\begin{array}{lll}
H_{o}: & \beta_{1}=0 \\
H_{1} & : & \beta_{1} \neq 0
\end{array}
$$

where $\beta_{1}$ is the coefficient of the first degree polynomial regression on the output and the order of the observation.

$$
W_{i}=\beta_{0}+\beta_{1} i+\varepsilon
$$

if $\beta_{1}$ is sufficiently different from zero, then the iid assumption is not held for the observations. The positive $\beta_{1}$ corresponds to the learning curve and the negative value corresponds to the fatigue curve (Leemis 2004).

For the observation collected from iid, the parameters can be estimated using Maximum Likelihood Estimators (MLE), Least Squares and the method of moments. MLEs are the most used in this paper due to special properties. MLEs for the normal distribution are

$$
\hat{\mu}_{i}=\bar{Y}_{i . .}=\frac{1}{R_{i}} \sum_{j=1}^{R_{i}} \bar{Y}_{i j .} \hat{\sigma}_{i}=\left(\frac{1}{R_{i}} \sum_{j=1}^{R_{i}}\left(\bar{Y}_{i j .}-\overline{\bar{Y}}_{i . .}\right)^{2}\right)^{1 / 2} .
$$

After a parameter is estimated, the statistical goodness of fit tests like Chi-squre and Kolmogorov-Smirnov are used to investigate the quality of the estimation. However, the statistical tests only provide advices. The effectiveness metamodel can also be justified by using test data. The results are often compared with outputs from corresponding simulation model. In this paper, the metamodel is validated by using the test data set.

If the parameter is estimated by MLEs. The following confidence interval can be used to test the effectiveness of the metamodel (Law and Kelton 2000): $I_{i}(\mu)=\hat{\mu}_{i} \pm \sigma_{i}(\mu)$ and $I_{i}(\sigma)=\hat{\sigma}_{i} \pm \delta_{i}(\sigma)$ where

$$
\delta_{i}(\eta)=\left(-E\left[\frac{\partial^{2} \ln L_{i}\left(\hat{\mu}_{i}, \hat{\sigma}_{i}\right)}{\partial \eta^{2}}\right]\right)^{-1 / 2}
$$

and

$\ln L_{i}\left(\mu_{i}, \sigma_{i}^{2}\right)=-R_{i} \ln \sqrt{2 \pi}-\frac{R_{i}}{2} \ln \sigma_{i}^{2}-\frac{1}{2 \sigma_{i}^{2}} \sum_{j=1}^{R_{i}}\left(\bar{Y}_{i j}-\mu_{i}\right)$. 
If the metamodel's output with the estimated parameters is within the confidence interval, the metamodel is not rejected. For example, if $95 \%$ of the experiment conditions are within the confidence interval then the metamodel is said to be not rejected.

$$
H_{0}: g_{1}\left(X_{i} ; \hat{\theta}_{\mu}\right) \in I_{i}(\mu) \cap g_{2}\left(X_{p} ; \hat{\theta}_{\sigma}\right) \in I_{i}(\sigma)
$$

Where $g_{1}$ and $g_{2}$ are the transformation function of mean and variance.

\section{SIMULATION AND RESULTS}

The purpose of simulation model is to gain insights the behavior of the supply chain so that the average total cost can be reduced. Thus the output here will be the average total cost. Many decision variables can be identified from Figure 3. We are interested in the initial inventory for each station, the selection of small $s$ and big $S$. We assume that the demand pattern at each location in the lowest echelon is the same. That is the demand interarrival time are the same across all demand locations. The demand interarrival time ranged from 0.1 to 2 corresponding to the busy and idle situation respectively. We divide the range into 10 smaller ranges at the following point $0.1,0.3,0.5,0.7,0.9,1.1,1.3$, $1.5,1.7,1.9$. Given the demand interarrival time, the initial inventory and the reorder level $s$ and order up to level $S$ need to be decided. Only after these variables are determined, the average total cost can be obtained by simulation. In other words, the final average total cost is affected by the demand interarrival time, the initial inventory, and the selection $s$ and $S$. The model was developed using Arena 10.0 and the data were analyzed using MATLAB 2000. Figure 5 shows the screenshot of a running model. For each experiment condition, we replicated for 100 times independently. For each replication, 2000 observations were collected. That is $N=10, R_{i}=100, K_{i j}=2000$. The simulation length is set to be 300 time units (day) to reach the steady output of the average total cost.

Figure 4 shows the relationship between the average total cost and the simulation time. At the beginning of the simulation, the fluctuation of the average total cost is large. Then it becomes much stabler. To better represent the simulation output, the data points at the beginning of each replication will be removed due to the high fluctuation. The Welch's moving average (Law and Kelton 2000) algorithm will be used to identify the cutoff point.

The initial inventory of each location is determined by using Equation (8).

$$
I=d(T+L)+z \sigma \sqrt{T+L}
$$

where $I$ is the calculated initial inventory; $d$ is the average daily demand. If the location is not in the lowest

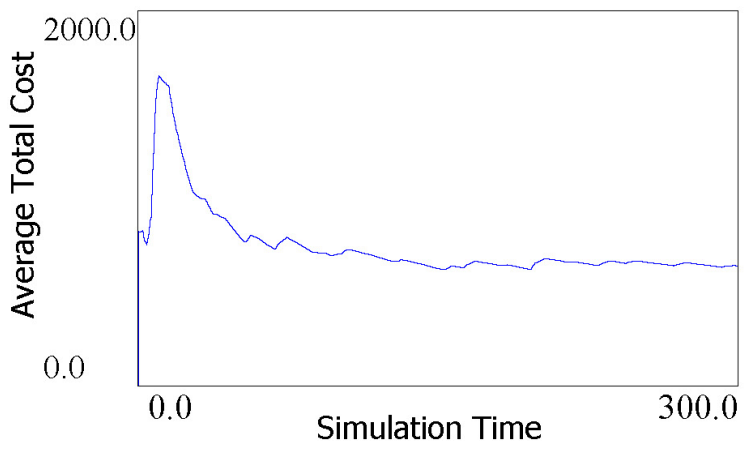

Figure 4: The average total cost versus the simulation time (demand interarrival time is 0.5 )

echelon then the demand will be the sum of all his children's demand; $T$ is the length of the review interval; $L$ is the lead time; $\sigma_{d}$ is the standard deviation of the demand per time period (for Poisson process, the variance is equal to the mean); $z$ is the safety factor (Sahin and Robinson 2006). $\Phi(z)=\frac{b}{b+h}$ where $b$ is the shortage cost and $h$ is the holding cost. In our model, we set $b=10, h=1$. For example, The calculation of the initial inventory of the location 2 given the demand rate is 0.5 is

$$
\begin{aligned}
d & =1 / 0.5+1 / 0.5+1 / 0.5=6 \\
T & =1 L=2 \\
\sigma_{d} & =6 \\
z & =1.335 \Phi(z)=\frac{10}{10+11} \\
Q & =6 *(1+2)+1.335 * 6 * \sqrt{1+2}=31.87
\end{aligned}
$$

We round the calculated value to the nearest integers towards infinity. The initial inventory for location 2 is 32 . Since the demand rate are the same across all locations in the lowest echelon, we assume the initial inventory is the same on each echelon. Note that Equation (8) is actually the order-up-to level of the periodic review system with on-hand inventory is zero. The main purpose of the initial inventory is to meet the service level agreement especially the immediately service level agreement (SLA0) at the beginning of the simulation. Location "7" and "8" have no requirement on immediate service, thus this calculation will tend to give more initial inventory then the real need. But we will simply assume they are the same since the difference are not big.

We calculate $S$ the same as initial inventory based on Equation (8). The $s$ is calculated using Equation (9) and rounded to the nearest integers towards infinity. 
Song, Li, and Garcia

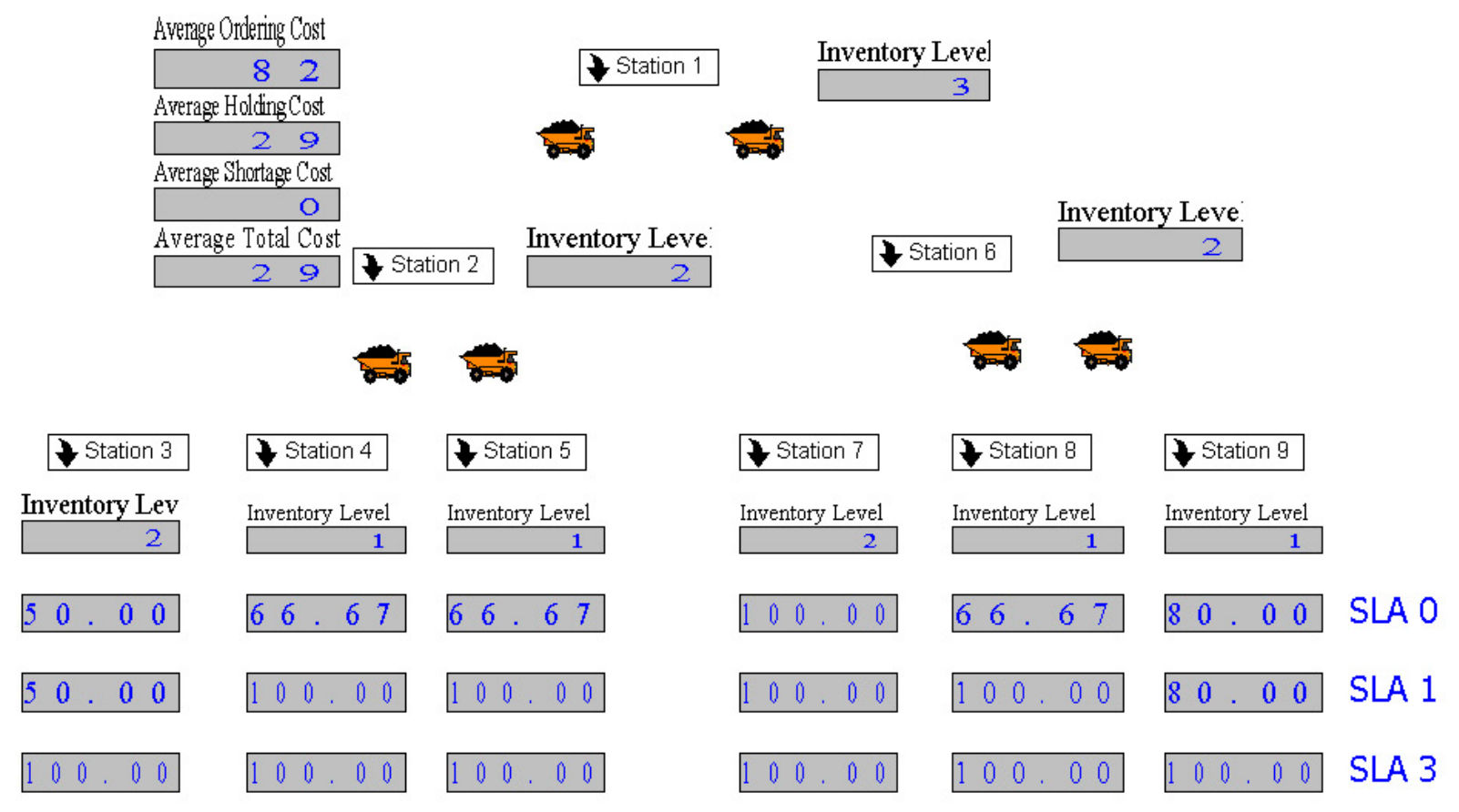

Figure 5: The screenshot of the running model

$$
s=d T+z \sigma \sqrt{T}
$$

We also assume that the $s$ and $S$ are the same on each echelon. That is, location 3,4,5,7,8,9 will have the same $s$ and $S$, while location 2 and 6 will have the same $s$ and $S$. Table 1 shows the the results of the initial inventory and the corresponding $s$ and $S$.The search for optimal $s$ and $S$ could also be done by using the application named OptQuest. But we found that the outputs of OptQuest are not reliable. The Service Level Agreement can not be maintained at all times if we run the simulation using the results of OptQuest.

We collected $N=10, R_{i}=100, K_{i} j=2000$ data to build our metamodel. Table 2 shows the Maximum Likelihood Estimations, the $\sigma_{i}(\eta)$. As it can be seen from the table, the value of $\beta_{1}$ is very close to 0 . That is the assumption (5) is valid for the collected data.

The polynomial linear regression algorithm is used to find the relationship between the experiment condition (mean demand inter arrival time) and the simulation output (average total cost). The degree of the polynomial regression is important. Higher degree will offer better fit but it tends to overfit. We will test the degree from 1 to 10 . The number of points will be recorded if the output of regression is out of the Maximum Likelihood Estimation Confidence Interval. The results are shown in Table 3. From the table, we know that the best polynomial degree of the mean average total
Table 1: The initial inventory level and the optimized $s$ and $S$. The subscript means the echelon level.

\begin{tabular}{crrrrrr}
\hline Demand & Inv & Inv & Inv & $s_{1}$ & $s_{2}$ & $s_{3}$ \\
interarrival time & $S_{1}$ & $S_{2}$ & $S_{3}$ & & & \\
\hline 0.1 & 557 & 160 & 39 & 141 & 71 & 24 \\
0.3 & 186 & 54 & 13 & 47 & 24 & 8 \\
0.5 & 112 & 32 & 8 & 29 & 15 & 5 \\
0.7 & 80 & 23 & 6 & 21 & 11 & 4 \\
0.9 & 62 & 18 & 5 & 16 & 8 & 3 \\
1.1 & 51 & 15 & 4 & 13 & 7 & 3 \\
1.3 & 43 & 13 & 3 & 11 & 6 & 2 \\
1.5 & 38 & 13 & 3 & 10 & 5 & 2 \\
1.7 & 33 & 10 & 3 & 9 & 5 & 2 \\
1.9 & 30 & 9 & 3 & 8 & 4 & 2 \\
\hline
\end{tabular}

cost is 9 and the best degree for the standard deviation is 5. Figure 6 and 7 show the estimated value of the mean and the standard deviation of the average total cost.

The result of the regression is shown in Table 4. Figure 8 and 9 show the value calculated from the metamodel and the value obtained through the simulation. As it can be seen from the figures, the predication is fairly accurate. Our metamodel shown in Table 4 is a good approximation of the real simulation model at the demand interarrival time range from 0.1 to 1.9. Figure 6 shows that at the end of this 
Table 2: Experiment condition, the MLE estimators, $\delta_{i}(\eta)$

\begin{tabular}{rrrrrrr}
\hline $\mathrm{i}$ & $X_{i}$ & $\beta_{1}$ & $\hat{\mu}_{i}$ & $\delta_{i}(\mu)$ & $\hat{\sigma}_{i}$ & $\delta_{p}(\sigma)$ \\
\hline 1 & 0.1 & 0.212 & 1710.750 & 7.286 & 36.538 & 5.208 \\
2 & 0.3 & -0.002 & 676.125 & 2.895 & 14.517 & 2.069 \\
3 & 0.5 & -0.065 & 463.519 & 2.214 & 11.101 & 1.582 \\
4 & 0.7 & -0.069 & 355.158 & 2.050 & 10.278 & 1.465 \\
5 & 0.9 & -0.034 & 324.532 & 1.612 & 8.084 & 1.152 \\
6 & 1.1 & -0.043 & 259.489 & 1.452 & 7.281 & 1.038 \\
7 & 1.3 & -0.047 & 249.443 & 1.436 & 7.200 & 1.026 \\
8 & 1.5 & -0.050 & 227.221 & 1.463 & 7.338 & 1.046 \\
9 & 1.7 & -0.030 & 205.267 & 1.344 & 6.742 & 0.961 \\
10 & 1.9 & -0.026 & 190.939 & 1.294 & 6.490 & 0.925 \\
\hline
\end{tabular}

Table 3: Number of rejected points versus the degree of the polynomial regression

\begin{tabular}{ccccccccccc}
\hline degree & 1 & 2 & 3 & 4 & 5 & 6 & 7 & 8 & 9 & 10 \\
\hline mean & 10 & 10 & 10 & 10 & 10 & 8 & 5 & 6 & 0 & 0 \\
std & 10 & 7 & 6 & 4 & 0 & 0 & 0 & 0 & 0 & 0 \\
\hline
\end{tabular}

range the metamodel can not accurately predict the mean of the average total cost. Large fluctuation can be observed there. This might indicate that a new metamodel is needed to study the behavior for range greater than 1.9.

\section{CONCLUSION}

The multi-echelon supply chain is intrinsically complicated. Due to the complexity and the randomness, it is extremely if not impossible to obtain a closed-form solution of optimal policy. Simulation can be used to study the multi-echelon supply chain. In this paper, we applied the metamodel methodolgy to study a multi-echelon supply chain with service level constraints.

Table 4: Polynomial Regression parameters (The Metamodel)

\begin{tabular}{rrr}
\hline & mean & stddev \\
\hline$X^{0}$ & 4764.809 & 57.425 \\
$X^{1}$ & -62224.032 & -261.499 \\
$X^{2}$ & 364832.866 & 549.541 \\
$X^{3}$ & -1184396.130 & -548.750 \\
$X^{4}$ & 2302665.093 & 257.470 \\
$X^{5}$ & -2787536.377 & -45.624 \\
$X^{6}$ & 2115542.596 & \\
$X^{7}$ & -977481.388 & \\
$X^{8}$ & 251096.506 & \\
$X^{9}$ & -27472.145 & \\
\hline
\end{tabular}

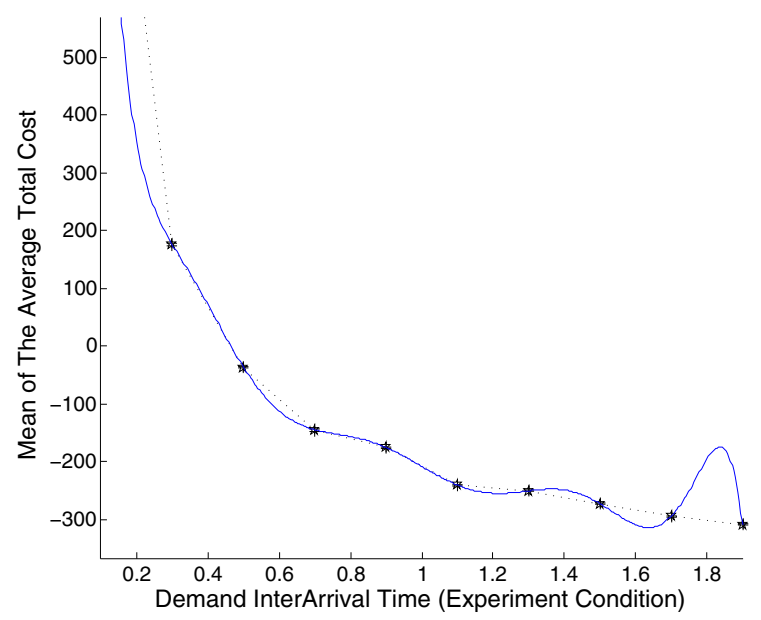

Figure 6: Estimated mean average total cost with the respective confidence intervals. The solid line is the polynomial fitting line with degree 9 .

Metamodels are abstractions of the simulation model that exposes the system's input-ouput relationship through simple mathematical functions. In our supply chain simulation model, the demand interarrival time is set to be the input variable and the average total cost is set to be the output variable. The goal is to reduce the average total cost as much as possible. To calculate the average total cost, the initial inventory and the $s$ and $S$ need to be calculated first. The initial inventory and $S$ are calculated according to the order-up-to level of the periodical review model. The values of $s$ is calculated based on the results of continuous review model.

The metamodel is tested by applying to the testing samples. The test results show that the metamodel performs well in the range from 0.1 to 1.9. The fitted closed-form expressions for the mean and standard deviation of average total cost are obtained. These expressions can be used as surrogate models to substitute the actual simulation model in its parent model.

The method can be applied to even more complex system. In this paper we only investigate the demand interarrival time as input. However, more input variables can be included so that the result metamodel can contain more information and more accurately approximate the real model.

\section{REFERENCES}

Axsater, S. 2000. Inventory control. Kluwer Academic Publishers.

Badiru, A. B., and D. B. Sieger. 1998. Neural network as a simulation metamodel in economic analysis of risky 
Song, Li, and Garcia

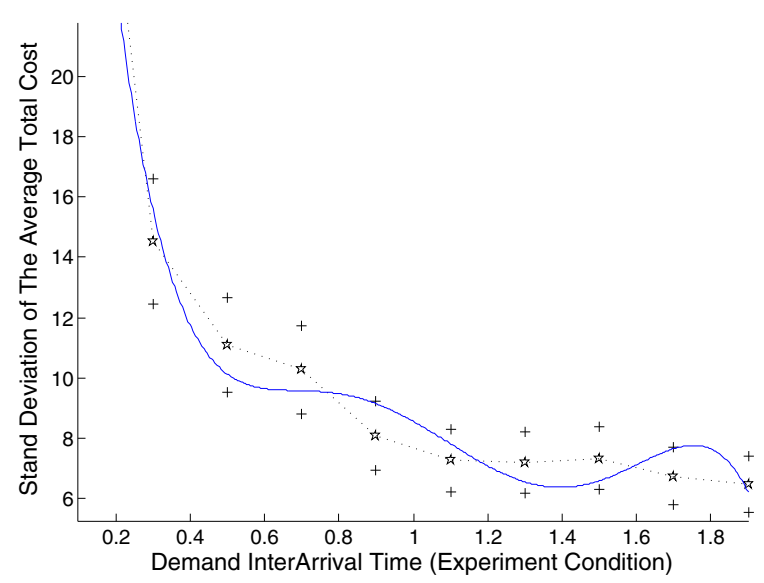

Figure 7: Estimated standard deviation of the average total cost with the respective confidence intervals. The solid line is the polynomial fitting line with degree 5 .

projects. European Journal of Operational Research 105 (1): 130-142.

Barton, R. R. 1992. Metamodels for simulation input-output relations. In Proceedings of the 1992 Winter Simulation Conference, 289-299. New York, NY, USA: ACM.

Caggiano, K., P. Jackson, J. Muchstadt, and J. Rappold. 2007. Optimizing service parts inventory in a multiechelon, multi-item supply chain with time-based customer service level agreements. Operations Research 55 (2): 303-318.

Caglar, D., C.-L. Li, and D. Simchi-Levi. 2004. Two-echelon spare parts inventory system subject to a service constraint. IIE Transactions 36 (7): 655-666.

Cheng, R. C. H. 1999. Regression metamodeling in simulation using bayesian methods. In Proceedings of the 1999 Winter Simulation Conference, ed. P. A. Farrington, H. B. Nembhard, D. T. Sturrock, and G. W. Evans, 330-335. New York, NY, USA: ACM.

Clark, A. J., and H. Scarf. 1960. Optimal policies for a multiechelon inventory problem. Management Science 6 (4): 475-490.

Hadley, G., and T. M. Whitin. 1985. Analysis of inventory systems. Prentice Hall.

Hopp, W., and M. Spearman. 2000. Factory physics. 2nd ed. McGraw-Hill Higher Education.

Kleijnen, J. P. 1986. Statistical tools for simulation practitioners. New York, NY, USA: Marcel Dekker, Inc.

Kleijnen, J. P. 2007a. Kriging metamodeling in simulation: A review. Discussion Paper 2007-13, Tilburg University, Center for Economic Research.

Kleijnen, J. P. 2007b. Regression models and experimental designs: a tutorial for simulation analysts. In Proceedings of the 39th conference on Winter simulation, ed. S. G. Henderson, B. Biller, M.-H. Hsieh, J. Shortle,

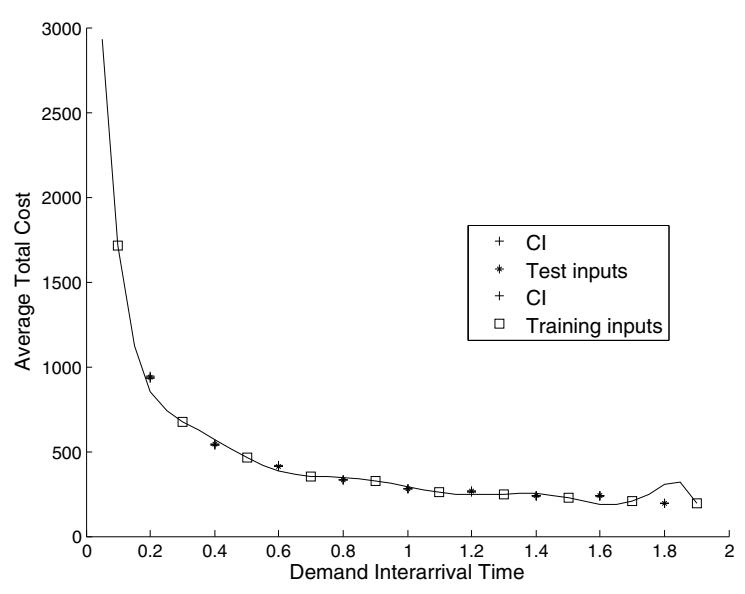

Figure 8: The predicted mean value on the test demand interarrival time versus the fitted polynomial line with degree 9.

J. D. Tew, and R. R. Barton, 183-194. Piscataway, NJ, USA: IEEE Press.

Kleijnen, J. P. 2008. Design and analysis of simulation experiments, Volume 111 of International Series in Operations Research \& Management Science. Springer. ISBN: 978-0-387-71812-5.

Kleijnen, J. P., and W. C. M. van Beers. 2005. Robustness of kriging when interpolating in random simulation with heterogeneous variances: Some experiments. European Journal of Operational Research 127 (3): 826-834.

Law, A., and W. Kelton. 2000. Simulation modeling and analysis. 3rd ed. McGraw-Hill.

Leemis, L. M. 2004. Building credible input models. In Proceedings of the 2004 Winter Simulation Conference, ed. R. G. Ingalls, M. D. Rossetti, J. S. Smith, and B. A. Peters, 29-40: Winter Simulation Conference.

Reis dos Santos, M. I., and A. M. Porta Nova. 2006. Statistical fitting and validation of non-linear simulation metamodels: A case study. European Journal of $\mathrm{Op}$ erational Research 127 (1): 53-63.

Sahin, F., and E. P. Robinson. 2006. Handbook of global logistics and supply chain management, Chapter Inventory Management in Global Supply Chains, 195. Sage Publications.

Santos, I. R., and P. R. Santos. 2007. Simulation metamodels for modeling ouput distribution parameters. In Proceedings of the 2007 Winter Simulation Conference, ed. S. G. Henderson, B. Biller, M.-H. Hsieh, J. Shortle, J. D. Tew, and R. R. Barton.

Silver, E. A., D. F. Pyke, and R. Peterson. 1998. Inventory management and production planning and scheduling. 3 ed. John Wiley \& Sons. ISBN-10: 0471119474. 
Song, Li, and Garcia

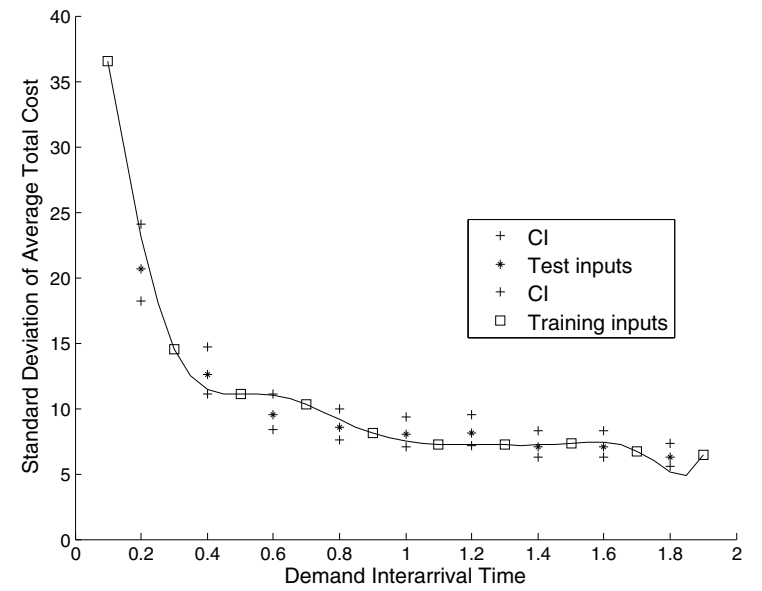

Figure 9: The predicted standard deviation value on the test demand interarrival time versus the fitted polynomial line with degree 5 .

Tijms, H. C. 1972. Analysis of (s,s) inventory models. Amsterdam, Mathematisch Centrum.

Wong, H., B. Kranenburg, G.-J. van Houtum, and D. Cattrysse. 2007. Efficient heuristics for two-echelon spare parts inventory systems with an aggregate mean waiting time constraint per local warehouse. OR Spectrum 29 (4): 699-722.

Zipkin, P. H. 2000. Foundations of inventory management. Irwin Professional Pub. ISBN-10: 0256113793.

\section{AUTHOR BIOGRAPHIES}

LAIGANG SONG is a PhD Student of Industrial and Information Engineering in the College of Engineering at the University of Tennessee. He got his master degree from the mechanical engineering in Chinese Academy of Sciences. His research interests lie in supply chain management, Complex systems modeling, simulation, and optimization. His email address for these proceedings is $\langle s l g @ u t k$. edu $>$.

XUEPING LI is an is an Assistant Professor of Industrial and Information Engineering and the Director of the Intelligent Information Engineering Systems Laboratory (IIESL) at the University of Tennessee - Knoxville. He holds a Ph.D. from Arizona State University. His research areas include information assurance, scheduling, web mining, supply chain management, lean manufacturing, and sensor networks. He is a member of IIE, IEEE and INFORMS. His research interests include: Quality of Service of computer and network systems and Information Systems Assurance, System Reliability, Complex systems modeling, simulation, and optimization, Scheduling, applied statistics, web mining, and Grid computing. His web addres is <http://web.utk.edu/ xli27> and his email address for these proceedings is <Xueping. Li@utk . edu>.

ALBERTO GARCIA is Professor and Associate Dean for Academic Affairs in College of Engineering at the University of Tennessee. He was a Professor of the Department of Industrial Engineering in Texas A\&M University before he came to the University of Tennessee. He received his $\mathrm{PhD}$ degree in industrial engineering from University of Illinois at Urbana-Champaign. His research includes Operations Research, Transportation and Logistics, Bridge and Pavement Management Systems, and Network Flow Optimization. He is a member of IIE and INFORMS. His web addres is $\langle$ http: //web. utk. edu/ ie/garcia.htm> and his email address for these proceedings is <agdeutk.edu> 